\title{
A Wave Texture Difference Method for Rainfall Detection Using X-Band Marine Radar
}

\author{
Lei Sun $\mathbb{D}^{1},{ }^{1}$ Zhizhong Lu $\mathbb{D},{ }^{1}$ Hui Wang $\mathbb{D},{ }^{2}$ Hong Liu $\mathbb{D}^{1},{ }^{1}$ and Xiuneng Shang $\mathbb{D}^{1}$ \\ ${ }^{1}$ College of Intelligent Systems Science and Engineering, Harbin Engineering University, No. 145 Nantong Street, \\ Harbin 150001, China \\ ${ }^{2}$ School of Electronics and Information, Jiangsu University of Science and Technology, Zhenjiang 212003, China \\ Correspondence should be addressed to Lei Sun; 371318826@hrbeu.edu.cn and Zhizhong Lu; luzhizhong@hrbeu.edu.cn
}

Received 12 December 2021; Revised 22 January 2022; Accepted 31 January 2022; Published 18 February 2022

Academic Editor: Antonio Lazaro

Copyright (c) 2022 Lei Sun et al. This is an open access article distributed under the Creative Commons Attribution License, which permits unrestricted use, distribution, and reproduction in any medium, provided the original work is properly cited.

To suppress the influence of rainfall when extracting sea surface wind and wave parameters using X-band marine radar and control the quality of the collected radar image, it is necessary to detect whether the radar image is contaminated by rainfall. Since the detection accuracy of the statistical characteristics methods (e.g., the zero pixel percentage method and the highclutter direction method) is limited and the threshold is difficult to determine, the machine learning methods (e.g., the support vector machine-based method and the neural network algorithm) are difficult to select appropriate quality and quantity of data for model training. Therefore, based on the feature that rainfall can change the sea surface texture, a wave texture difference method for rainfall detection is proposed in this paper. Considering the spatial rainfall is uneven, the polar coordinates of the radar image are converted into Cartesian coordinates to detect rainfall. To express the maximum wave difference more accurately, the calculation method of the pixels in the radar texture difference map is redefined. Then, a consecutive pixel method is used to detect the calculated radar texture difference map, and this method can detect adaptively with the change of wind. The data collected from the shore of Haitan Island along the East China Sea are used to validate the effectiveness of the proposed method. Compared with the zero pixel percentage method and the support vector machine-based method, the experimental results demonstrate that the proposed method has better rainfall detection performance. In addition, the research on the applicability of the proposed method shows that the wave texture difference method can finish the task of rainfall detection in most marine environments.

\section{Introduction}

Currently, X-band marine radar has become one of the most widely used marine remote sensors. Generally, X-band marine radar is installed on the ship, which is used for ship positioning, navigation avoidance, and narrow channel navigation [1-3]. The wind parameters, such as wind speed and direction, and the wave parameters, such as wave height, direction, and period, can be extracted from the acquired radar images $[4,5]$. Although remote sensing measurement has many advantages, such as convenient use, large measurement range, and real-time information feedback, various noises often exist in the acquired radar images.

These common noises come from cochannel interference, background, and rainfall. The cochannel interference and back- ground noise are relatively slight, which can be suppressed by algorithms $[6,7]$. The rainfall will change the roughness of sea surface and destroy the texture feature of sea wave [8]. Heavy rainfall will seriously affect the propagation of electromagnetic wave and make it difficult to reach the sea surface, which means that the sea surface cannot be normally observed by radar systems $[9,10]$. Meanwhile, the sea clutter will be mixed with rain clutter under heavy rainfall, which presents high brightness echo and wide range fuzzy noise in the radar image. Since the backscatter mechanism of radar is complex in the presence of rainfall, it is difficult to eliminate the rainfall interference from radar images [11]. In order to avoid affecting the accuracy of the wave parameter extraction, these radar images should be discarded $[12,13]$. Therefore, to control the quality of the radar image, it is necessary to detect whether the radar image is 
contaminated by rainfall before extracting the wave parameters of the radar image $[14,15]$. Currently, this research has attracted the attention of many researchers and has achieved many representative achievements.

In the early years, the statistical characteristic method is used to detect whether the radar image is contaminated by rainfall, but this method can only reflect the local feature rather than the overall feature $[16,17]$. The concept of zero pixel percentage (ZPP) was first proposed by Lund et al. [18]. The ratio of the number of image pixels with zero intensity to the overall number of pixels is identified as a quality control parameter, and the threshold is set to $50 \%$ to determine the raincontaminated radar image. Although the rain-contaminated radar image can be successfully detected, the setting of the threshold needs to be further studied. The effectiveness of the ZPP method is verified in [19], and the threshold of rainfall detection is reset to improve the detection accuracy. Two sets of radar systems, Furuno and Decca, were used for the experiments. The experimental results show that the radar image is regarded as rain-contaminated radar image when the ZPP of the radar image is less than $20 \%$ for the Furuno radar or $10 \%$ for the Decca Radar.

Additionally, the improved ZPP method and new rainfall detection method are also proposed. A method called highclutter direction (HCD) is proposed. For a radar image, if the intensity of all pixels in an azimuth direction is greater than a certain value, the direction is defined as a high-clutter direction. If the ratio of the high-clutter direction to all directions is greater than $5 \%$, the radar image is regarded as a raincontaminated radar image [20, 21]. In [22], an image gray value method is proposed to identify whether the radar image is contaminated by rainfall, and a large number of experiments are used to determine the gray value threshold. The threshold of 0.55 is chosen, i.e., the gray value is less than 0.55 if the radar image is contaminated by rainfall; otherwise, the gray value is greater than 0.55 . A method based on support vector machine (SVM) is proposed to detect rainfall from X-band marine radar images in [23]. However, the performance of the detection model needs to be further improved under the swelldominated sea, and the effectiveness of the SVM-based method needs to be further verified. Based on the sea clutter analysis method proposed by Dong and Merrett [24], the correlation coefficient difference (CCD) method is proposed to detect rainfall from $\mathrm{X}$-band marine radar images, and the detection threshold is set to $1 /$ e. Since the correlation coefficient in the azimuth direction between light rain and heavy rain has little difference, and a correlation difference fluctuation exists in the experiment, the performance of the CCD method needs to be further studied [25]. In [26], an unsupervised clustering-based method is proposed to identify lowbackscatter and rain-contaminated regions in X-band marine radar images. According to the distinct difference in texture and pixel intensity distribution between low-backscatter, rain-free, and rain-contaminated, four types of features are extracted and combined into feature vectors, which are mapped into $10 \times 10$-neuron self-organizing map (SOM). The hierarchical agglomerative clustering algorithm is used to identify the regions of rain-free, rain-contaminated, and low-backscatter in X-band marine radar images. Because the unsupervised clustering-based method is relatively new, the effectiveness of the algorithm needs more data validation in the future.

$\mathrm{Lu}$ et al. focus on the sea wave research by using X-band marine radar. On the research of rainfall, a classical analysis method of rainfall observation data is proposed. The statistical characteristic - mean value and variation coefficient of a radar echo image-is used as classification standard of the rain-free and rain-contaminated radar images for rainfall detection, and this method has been widely used in the field of radar image rainfall detection [16]. Additionally, the rainfall detection area is defined in the occlusion area, and the ratio of zero intensity to echo (RZE) method for rainfall detection is proposed. The detection threshold is determined to improve the accuracy of rainfall detection by statistical analysis of a large number of radar image data, and it is proposed for the first time to retrieve the rainfall intensity level from the determined raincontaminated radar image. Currently, the RZE method has been published and widely recognized [27].

In recent years, the texture features of images are extracted to recognize and classify these images, which are widely used in various fields and gradually become a hot research topic. In [28], a semantic segmentation method of radar image based on key superpixels and texture complexity analysis is proposed to divide the landform. The results show that the algorithm can distinguish different landforms more effectively, suppress the influence of noise, and achieve the semantic segmentation of radar image. In [29], the incident angle (IA) dependence of seven commonly used grey level cooccurrence matrix (GLCM) texture features is investigated, and the results are included in the Gaussian incident angle (GIA) classifier for evaluation. In $[30,31]$, a new method of the image texture statistics and analysis is proposed by using different texture features of undamaged buildings and collapsed buildings. The proposed method is used to extract the building damage information for effective emergency decision-making.

Since the sea wave usually has certain texture information, and the existence of rainfall will change the texture information of the sea wave, the change of the information can be used to identify the rain-contaminated radar image. A simple scheme is introduced in [32] to identify the rain-contaminated portion in such an image, which ensures that the less-affected portions may still be useful for sea surface parameters extraction. Different from the method of the texture feature classification and statistics, this scheme is based on spatial variability analysis to effectively identify whether the azimuthal direction of the radar image is rain-contaminated by rainfall. However, the single radial radar line can only represent the rainfall inhomogeneity in the azimuthal direction but not in the spatial radial direction. Moreover, the adjacent $3 \times 3$ space matrix cannot represent the wave feature when the radial resolution of the radar is $7.5 \mathrm{~m}$. To improve the accuracy of rainfall detection, a wave texture difference (WTD) method is proposed in this paper. Considering the spatial rainfall is uneven, the polar coordinates of the radar image are converted into Cartesian coordinates to detect rainfall. To express the maximum wave difference more accurately, the calculation method of the pixels in the radar texture difference map is redefined. This article is the first article to systematically and comprehensively study rainfall detection by 


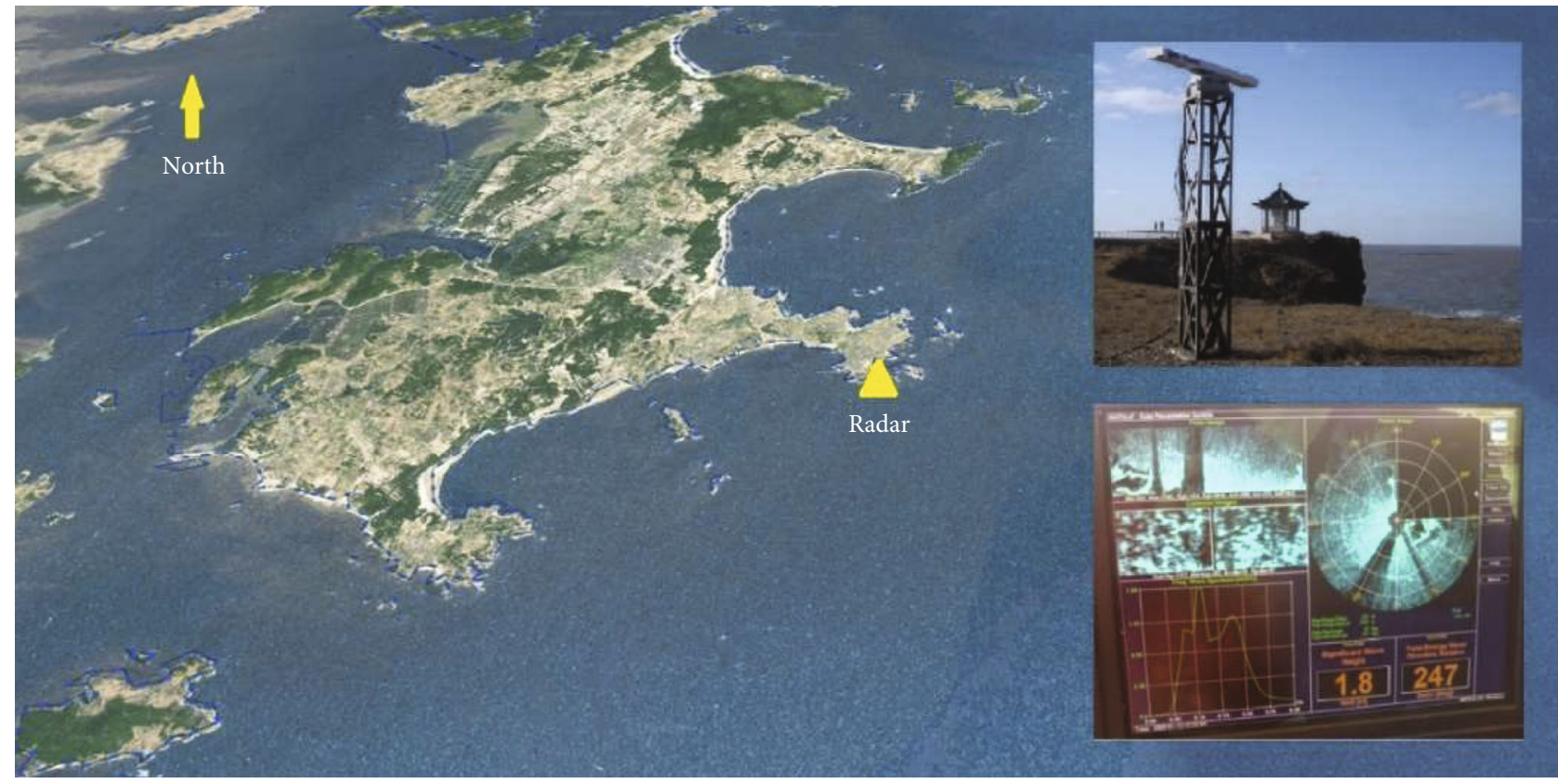

FIgURE 1: The experimental site and radar equipment.

using X-band marine radar texture difference map. In addition, the detection accuracy of the proposed method under different wave heights is also discussed.

This article describes a WTD method to detect rainfall from $\mathrm{X}$-band marine radar texture difference map. Data overview and image preprocessing are described in Section 2. Section 3 reviews the rainfall detection method based on the azimuthal direction in the texture map. Section 4 presents the process of rainfall detection based on the proposed method. In Section 5, the effectiveness and applicability of the proposed method are certified based on the acquired X-band marine radar image data. Conclusions and outlook are given in Section 6.

\section{Data Overview and Image Preprocessing}

The collected radar image data cannot be directly used to detect rainfall and should be pre-processed. The cochannel interference of the collected radar image is suppressed, and then the appropriate rainfall detection area is selected. The Cartesian diagram obtained by the nearest neighbor interpolation method is used for rainfall detection.

2.1. Data Overview. From 2013 to 2015, the wave monitoring equipment developed by the research group of Harbin Engineering University was used to collect data on the shore of Haitan Island along the East China Sea, and the position was $25^{\circ} 27^{\prime} \mathrm{N}$ and $119^{\circ} 50^{\prime} \mathrm{E}$. The radar image sequence collected by the wave monitoring equipment consisted of 32 radar images. The collected data is digitized by a 14-bit high-speed acquisition card, and the digitized backscatter intensities ranging from 0 to 8192 . A large number of measured data under different weather and sea conditions were saved during this period, and the data used for the present work were collected during this period. The experimental site and applied radar equipment are shown in Figure 1.
TABLE 1: The configuration of the X-band marine radar.

\begin{tabular}{lc}
\hline Radar parameters & Value \\
\hline Electromagnetic wave frequency & $9320 \sim 9500 \mathrm{MHz}$ \\
Pulse power & $25 \mathrm{~kW}$ \\
Radar antenna rotational speed & $26 \mathrm{r} / \mathrm{min}$ \\
Antenna gain & $31 \mathrm{~dB}$ \\
Polarization & $\mathrm{HH}$ \\
Horizontal beam width & $0.9^{\circ}$ \\
Vertical beam width & $21^{\circ}$ \\
Pulse repetition frequency & $2000 \mathrm{~Hz}$ \\
Pulse width & $0.07 \mu \mathrm{s}$ \\
Grazing angle & $<5^{\circ}$ \\
Range resolution & $7.5 \mathrm{~m}$ \\
\hline
\end{tabular}

The radar image data used in this paper were collected from August 2013, and the configuration of the X-band marine radar is given in Table 1 . The height of the radar antenna is $45 \mathrm{~m}$ and the rotation period is about $2.7 \mathrm{~s}$. The radar image resolution is $7.5 \mathrm{~m}$ in distance and $0.1^{\circ}$ in azimuth, covering an area with a radius of $4300 \mathrm{~m}$.

The rain gauge recorded synchronously in minutes with an accuracy of $0.1 \mathrm{~mm}$. Referring to the actual radar image, the micro rainfall less than $0.1 \mathrm{~mm}$ was recorded as $0.05 \mathrm{~mm}$. The wave buoy used in this paper was deployed about $800 \mathrm{~m}$ from the radar, and the water depth of the buoy location was about $25 \mathrm{~m}$. The buoy measured the wave height as the ground truth, with a sampling frequency of $2 \mathrm{~Hz}$.

2.2. Cochannel Interference Suppression. The cochannel interference often exists in the acquired radar image, which seriously affects the quality of the radar image. Figure 2 shows a 


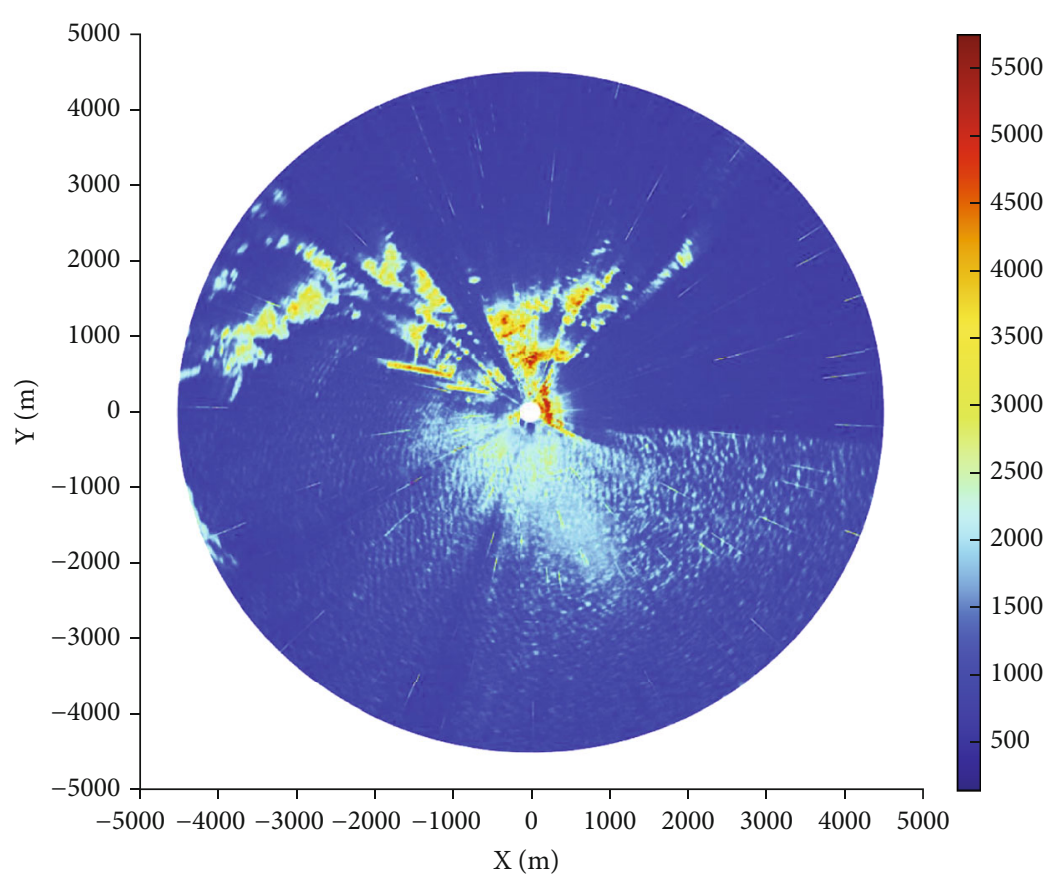

FIgURE 2: Raw radar image with cochannel interference.

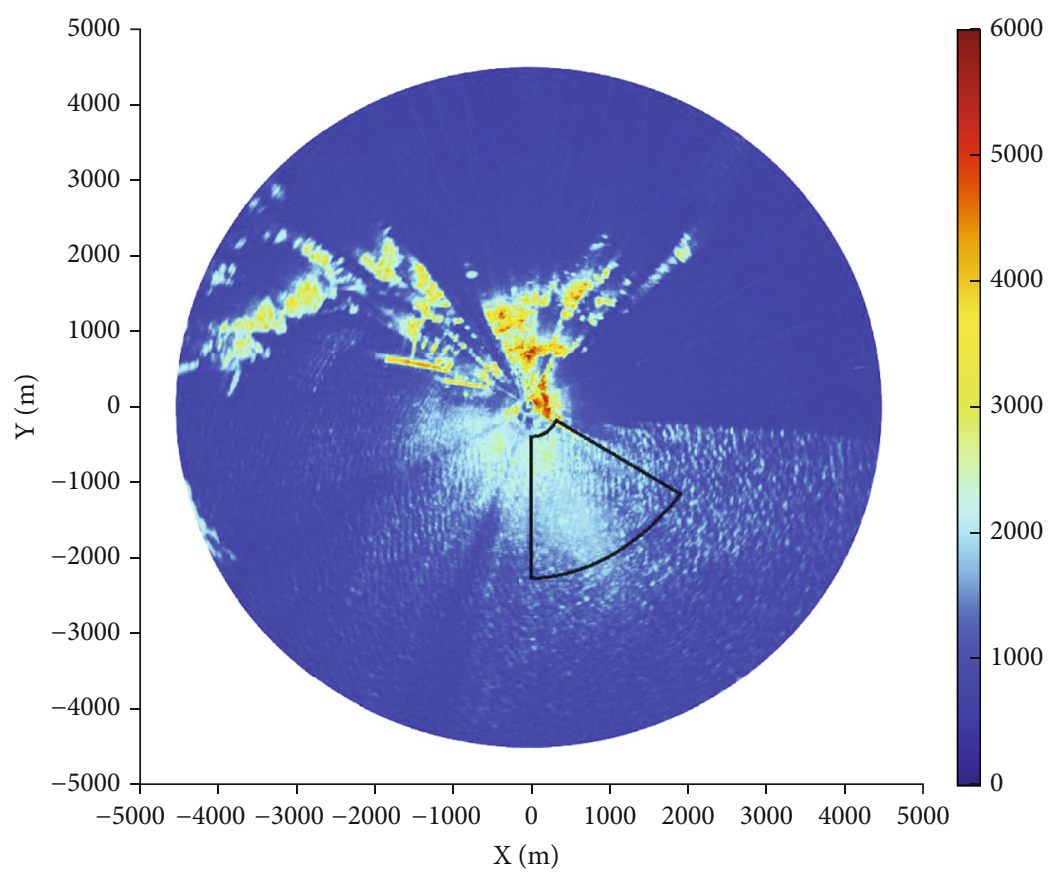

FIGURE 3: Figure 2 after cochannel interference removal .

radar image collected on August 21, 2013. The colorbar represents the digitized range value $(0 \sim 8192)$ of radar backscatter. Since cochannel interference exists in this image, it is necessary to suppress cochannel interference before detecting rainfall. According to the spatial correlation property of sea clutter in the X-band marine radar images, a method for detecting cochannel interference noise is proposed [6]. Firstly, an appro- priate threshold is determined by the constant false alarm rate method, and the correlation coefficient method is used to detect the specific location of the interference noise lines in the radar image. Then, the modified Laplace linear template is adopted to locate the interference noise points, and the detected noise points are repaired by the segmental interpolation method to filter out the noise. 


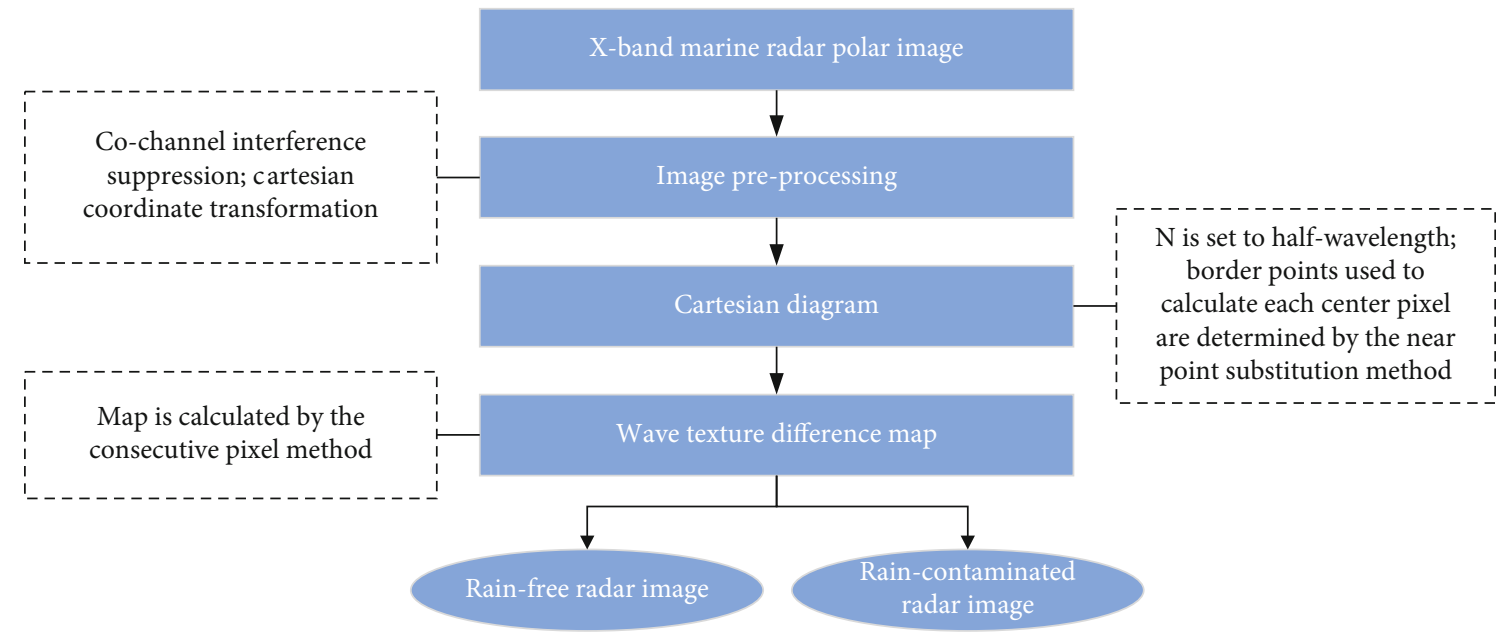

FIgURE 4: The flowchart of rainfall detection based on the WTD method.

2.3. The Selection of the Rainfall Detection Area. Figure 3 shows Figure 2 after cochannel interference suppression. Since the random superposition interference of various echo noises such as fixed objects and ships exists in the whole radar image, which affects the accuracy of rainfall detection, it is necessary to select the appropriate area in the radar image for rainfall detection [25].

It is assumed that the spatial distribution of rainfall is uniform in a short period of time. Considering the influence of topography in the observation area, the radial distance from $240 \mathrm{~m}$ to $2160 \mathrm{~m}$ and azimuth starting at $125^{\circ}$ in the radar image is selected. The selected analysis area is denoted by the black solid line.

Then, the pixel intensity values of the selected area are mapped into the Cartesian coordinate system by using the nearest neighbor interpolation method to obtain the corresponding Cartesian diagram. The calculation equation of the nearest neighbor interpolation can be described as follows:

$$
\left\{x_{0}=r \times \cos \theta, y_{0}=r \times \sin \theta,\right.
$$

where $r$ is the distance between a certain point and the center in the radar image, and $\theta$ is the angle from the bow direction. Equation (1) denotes that the point $(r, \theta)$ in polar coordinates is mapped to the point $\left(x_{0}, y_{0}\right)$ in Cartesian coordinates.

To preserve the wave feature to the maximum extent, a Cartesian diagram generally contains $8 \sim 12$ complete wavelengths of sea waves. The Cartesian diagram is $256 \times 256$ pixels $(1920 \mathrm{~m} \times 1920 \mathrm{~m})$, and the corresponding calculated radar texture difference map is also $256 \times 256$ pixels.

\section{The Rainfall Detection Method Based on the Azimuthal Direction in the Texture Map}

In this section, the rainfall detection method based on the azimuthal direction in the texture map is briefly reviewed. The calculation method of the texture map is introduced, and a rainfall detection scheme is used to determine whether the obtained texture map is contaminated by rainfall.

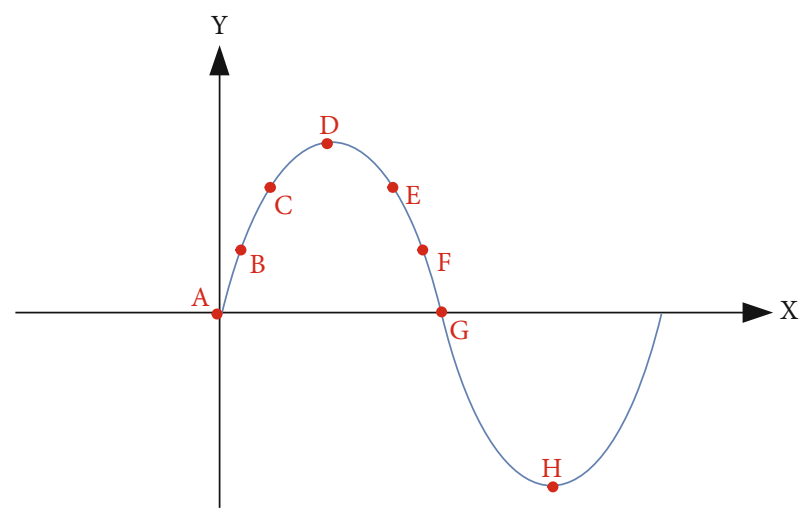

FIgURE 5: The representative position pixels in a complete wavelength of sea wave.

3.1. The Texture Map is Calculated by Root-Mean-Square Difference. In [32], the backscatter signal of the X-band marine radar is passed through a logarithmic amplifier before being converted to gray-scale image. Each pixel of the gray-scale image is calculated by the Equation of rootmean-square (RMS) difference, and the texture map used to quantify the spatial variability of the intensity is obtained. In order to improve the readability of the image, the pixel intensities of the texture map are also rescaled to the same gray scale (i.e., $[0,255]$ here) as that of the radar image. The RMS difference can be expressed as

$$
T_{a, b}=\sqrt{\frac{1}{9}} \sum_{i=-1}^{i=1} \sum_{j=-1}^{j=1}\left(I_{a, b}-I_{a+i, b+j}\right)^{2}
$$

where $I_{a, b}$ denotes the image intensity of pixel $(a, b)$ in the range $(a)$ and azimuth $(b)$, and $I_{a+i, b+j}$ denotes the image intensity of pixel $(a+i, b+j)$ in the range $(a+i)$ and azimuth $(b+j)$. The texture intensity of the pixel $(a, b)$ is computed in the polar domain by the RMS difference of the intensity levels of this pixel and its eight adjacent pixels. 
3.2. Rainfall Detection in the Azimuthal Direction of the Texture Map. A scheme is proposed in [32]. For each azimuthal direction in the obtained texture map, when the number of pixels with intensity greater than the threshold $I_{T}$ (the initial $I_{T}$ is set to 40) is less than a specified value $N_{G}$ (this value is 20 in [32]), the azimuthal direction is identified as rain-contaminated direction, and the radar image is determined as rain-contaminated radar image. Since the pixel intensity of the image increases with wind speed, the number of pixels with intensity higher than $I_{T}$ will also increase. Therefore, the threshold $I_{T}$ needs to be determined adaptively with the wind speed.

\section{Rainfall Detection Based on the WTD Method}

For the rainfall detection method proposed in Section 3, this section improves the method and proposes a new rainfall detection method based on the WTD. Firstly, the acquired radar image cannot be directly used for rainfall detection and should be preprocessed. The rainfall detection area is selected in the radar image after suppressing cochannel interference to obtain the Cartesian diagram by using the coordinate transformation method. Then, the maximum texture difference in the sea wave is discussed, and all the pixels in the Cartesian diagram are calculated to obtain the corresponding radar texture difference map. Finally, a consecutive pixel method is used to detect rainfall in radar texture difference map. The flowchart of the proposed rainfall detection method is presented in Figure 4, and the detailed implementation process is as follows.

4.1. The Calculation Method of the Radar Texture Difference Map. In Section 3.1, the texture intensity of the pixel $(a, b)$ is computed in the polar domain by the RMS difference of the intensity levels of this pixel and its eight adjacent pixels, which is not enough to express the wave difference feature with the wavelength of $60 \sim 150 \mathrm{~m}$. Therefore, the Nth border point used to calculate the pixel $(a, b)$ is further discussed, and the value of $N$ is reset.

The blue curve in Figure 5 shows a complete wavelength of sea wave, and the eight points (point $A$ to point $H$ ) are the pixels with representative positions on the wave curve. The points $D$ and $H$ are the crest and trough of the wave, respectively, and the points $A$ and $G$ are the zero points of the wave curve. The points $B$ and $C$ divide the curve $\mathrm{AD}$ into three equal parts, and the points $E$ and $F$ divide the curve DG into three equal parts. In the process of obtaining the radar texture difference map, in order to reflect the maximum texture difference of any pixel on each wave curve, the $N$ is set to $1 / 4$ wavelength, half-wavelength, and full-wavelength for analysis, respectively. When the points $A$ and $G$ on the wave curve are calculated, the maximum texture difference is the points $D$ and $H$, and the $N$ can be set to $1 / 4$ wavelength. When the point $B$ or point $C$ on the wave curve is calculated, the maximum texture difference is the point $H$. Therefore, the $1 / 4$ wavelength (point $E$ and point $F$ ) is not enough to express the maximum texture difference, and the $N$ should be increased. When the point $D$ on the wave curve is calculated,

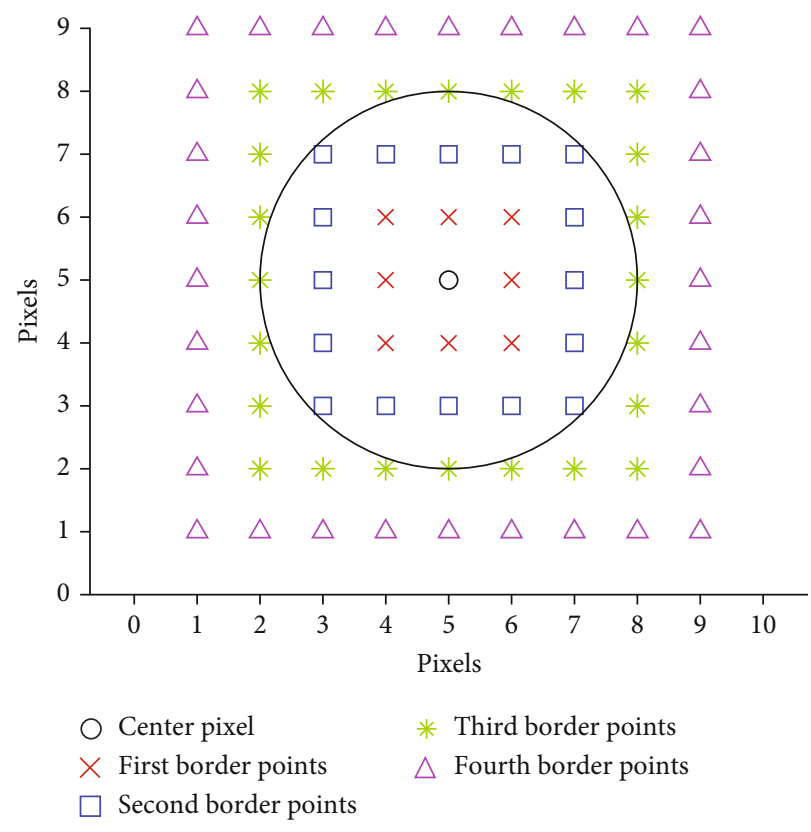

FIgURE 6: The distance distribution of the pixels.

the maximum texture difference is the point $H$, which is the maximum texture difference on the whole wave curve, and the $N$ can be set to half-wavelength. When the $N$ is set to be greater than half-wavelength, the maximum texture difference on the wave curve cannot be expressed. Therefore, when the $N$ is set to half-wavelength, the texture difference of the calculated radar texture difference map can be expressed most clearly.

Since the wavelength of sea wave is about $60 \sim 150 \mathrm{~m}$ and the radar distance resolution is $7.5 \mathrm{~m}$, the half-wavelength of the maximum wavelength is selected. The $N$ is set to $75 / 7.5=10$.

The set $N$ is used to calculate the texture difference map of the X-band marine radar, and the distance distribution of the pixels is shown in Figure 6. The black circle point is the center pixel to be calculated, and the red cross points adjacent to the center pixel are the first border points. The blue square points, green star points, and pink triangle points are the second, third, and fourth border points, respectively. If the third border points are used to calculate the center pixel, it can be found that the distance between the points in the directions of $0^{\circ}, 90^{\circ}$, $180^{\circ}$, and $270^{\circ}$ on the third border points and the center pixel is $3 \times 7.5 \mathrm{~m}$, and the distance in other directions is greater than $3 \times 7.5 \mathrm{~m}$. In addition, the distance between the points in the directions of $45^{\circ}, 135^{\circ}, 225^{\circ}$, and $315^{\circ}$ on the second border points and the center pixel is closer to $3 \times 7.5 \mathrm{~m}$. Therefore, the border points used to calculate the center pixel are determined by the near point substitution method in this article, and the steps of this method are as follows:

(1) The number of the pixels on the $N$-th border point is $l$, where $l=(2 \times \mathrm{N}+1) \times 4-4$. It should be noted that the number of the border points needed to calculate the center pixel increases with the increase of the N. After analysis, the border points needing to be included in the calculation of center pixel are 


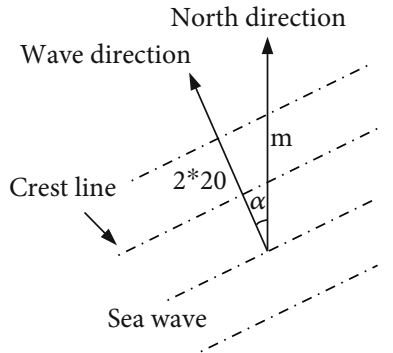

(a) $0 \leq \alpha<\pi / 4$

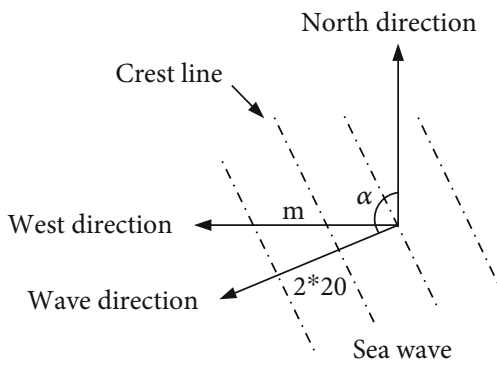

(c) $\pi / 2 \leq \alpha<3 \pi / 4$

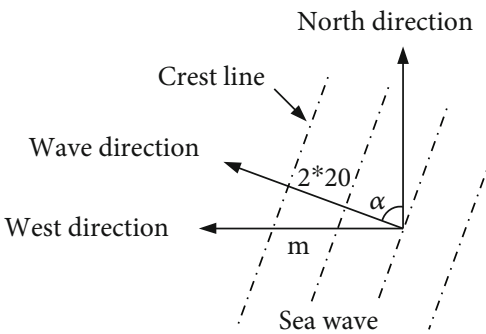

(b) $\pi / 4 \leq \alpha<\pi / 2$

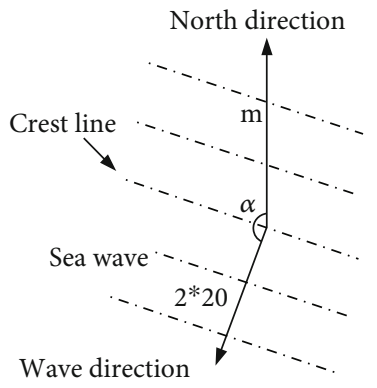

(d) $3 \pi / 4 \leq \alpha<\pi$

FIgURE 7: The calculation method of the $m$ consecutive pixels: (a) $0 \leq \alpha<\pi / 4$, (b) $\pi / 4 \leq \alpha<\pi / 2$, (c) $\pi / 2 \leq \alpha<3 \pi / 4$, and (d) $3 \pi / 4 \leq \alpha<\pi$.

$\{N, N-1,1 \leq N<6, N, N-1, N-2,6 \leq N<9, N, N-1, N-2, N-3,9 \leq N<12, N, N-1, N-2, N-3, N-4,12 \leq N<15 . \vdots$

(2) The radial distances between the border points determined by Equation (3) and the center pixel are calculated, respectively. The first $n$ points with the smallest difference between the radial distance and the $N \times 7.5 \mathrm{~m}$ are used to calculate the wave texture difference value. To satisfy the $l$ pixels to calculate the center pixel, here $n$ takes the value of $l$

(3) The center pixel is calculated by the first $n$ selected points, and the calculation method can be described as

$$
\frac{T_{a, b}=\sqrt{\sum_{i=1}^{n}\left(I_{a, b}-I_{i}\right)^{2}}}{n,}
$$

where $T_{a, b}$ is the texture difference value of the pixel $(a, b)$ in the radar texture difference map, $I_{a, b}$ and $I_{i}$ are the pixel intensities of the center pixel $(a, b)$ and the $n$ selected points in the Cartesian diagram, and the value range is $0 \sim 255$.

Since the $N$ is set to 10 , the radial distances between the seventh to tenth border points and the center pixel need to be calculated. Among the 272 selected points, the first 80 points with the smallest difference between the radial distance and $10 \times 7.5 \mathrm{~m}$ are used to calculate the wave texture difference value. All the pixels in the Cartesian diagram are calculated by Equation (4) to obtain the corresponding radar texture difference map.

4.2. Rainfall Detection from the Radar Texture Difference Map. A consecutive pixel method is used to detect rainfall from radar texture difference map. Each column (row) of the radar texture difference map is detected, and 256 detection results are obtained. The detection method for each column (row) is that if the intensity of $m$ consecutive pixels is within a certain range, this column (row) is identified as rain-contaminated. Since the rows and columns of the radar texture difference map are equivalent, this map is determined as rain-contaminated when $m$ consecutive detection results in the obtained 256 detection results are identified as rain-contaminated. The corresponding radar image is rain-contaminated radar image.

When the radar image is not contaminated by rainfall, the size of the wave texture mainly depends on the wave height and wind. Therefore, a large number of rain-free radar images under different wave heights are selected to calculate the radar texture difference map. The median values of all pixel intensities in each calculated texture difference map are statistically analyzed, and the average value of these median values is determined as $T_{H} \cdot T_{H}$ is an initial pixel threshold for the initial count of the number of pixels. When 


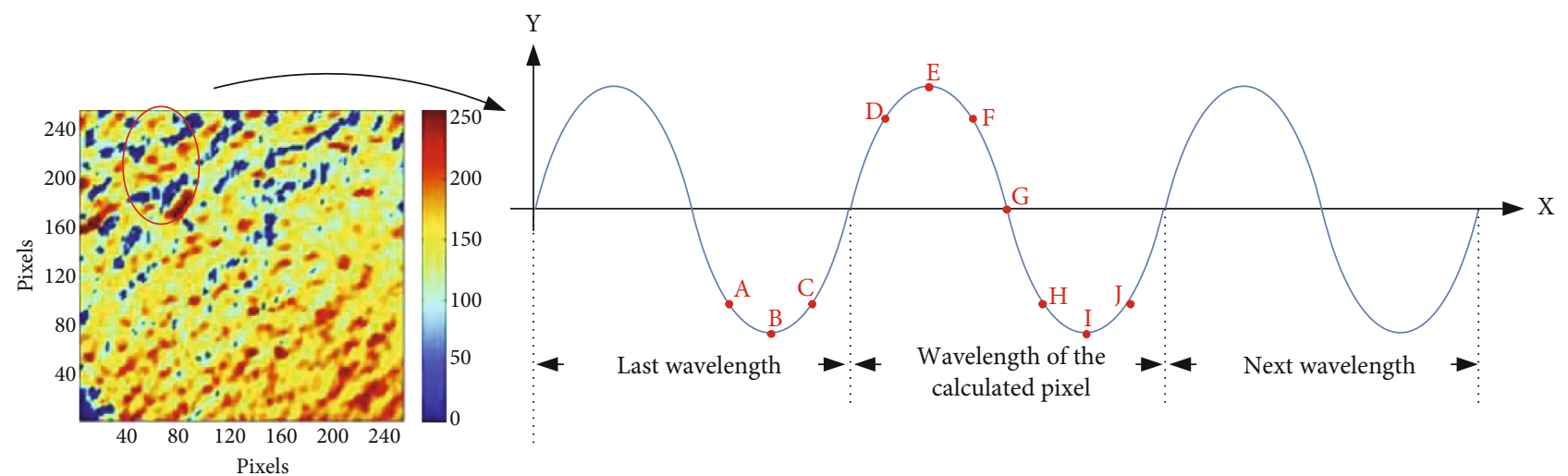

FIgURE 8: The wavelengths of the three adjacent waves in a Cartesian diagram.

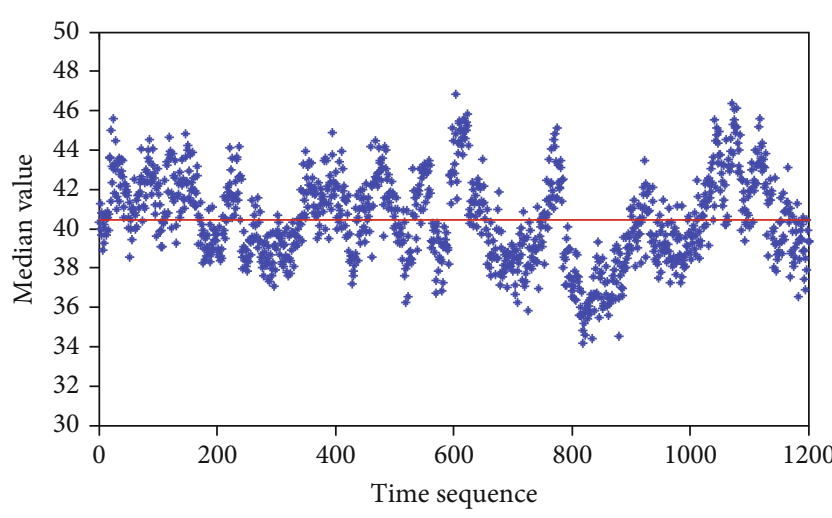

- Average value

* Median value

FIgure 9: Determination of the $T_{H}$.

the radar image is contaminated by rainfall, the wave texture will be smoothed, and the wave texture difference will be reduced. The overall pixel intensity of the obtained texture difference map decreases, and the number of pixels in $\left[0, T_{H}\right]$ increases. Due to the effect of wind, the overall pixel intensity of the radar texture difference map will change in a small range. Therefore, the certain range in the detection of each column (row) of the map is defined as $\left[k, k+T_{H}\right], k=0,1,2, \cdots$ , 256- $T_{H}$. After a large number of experiments, it is found that most of the pixels in the radar texture difference map are low pixels. Hence, $0 \leq k \leq 10$ in most experiments.

In addition, the selection of the number of consecutive pixels as well as whether the radar texture difference map is detected row-by-row or column-by-column is determined by the angle relationship between the wave direction and the north direction. From Figure 7, the dotted line is the crest line, and a complete wavelength is between the two crest lines. $\alpha$ is the angle between the wave direction and the north direction, and 20 pixels denote the number of pixels of a complete wavelength in the wave direction. Since the value of the wave direction can be known in advance, the $\alpha$ can be determined. The following factors need to be considered for the determination of consecutive pixel $\mathrm{m}$ :

(1) Since the border points used for the center pixel calculation are based on the information of two neighboring wavelengths when the Cartesian diagram is calculated by using half-wavelength distance, each pixel of the calculated radar texture difference map also contains the corresponding information. Figure 8 shows the wavelengths of the three adjacent waves in a Cartesian diagram. When the texture difference of the point $D$ is calculated by Equation (4), the border points used for the calculation contain the point $A$ and point $H$, and the point $A$ is a pixel within the last wavelength. The border points used to calculate the point $F$ contain the point $C$ and point $J$, and the point $C$ is a pixel within the last wavelength. Similarly, the border points used for the calculation of the point $H$ and point $J$ contain the pixels within the next wavelength. Thus, each pixel of the radar texture difference map contains the information of two neighboring wavelengths

(2) System delays and errors often affect the acquisition of radar images, which makes the wavelength range of sea wave in the acquired radar images often larger than the actual wavelength range (slightly greater than $60 \sim 150 \mathrm{~m})$. When the consecutive pixel $m$ is set to the number of pixels of one wavelength, it is difficult to accurately express the spatial rainfall in the radar texture difference map using the consecutive pixel method

Therefore, the continuous pixel $m$ is set to the number of pixels of two wavelengths. The $m$ is defined as follows: 


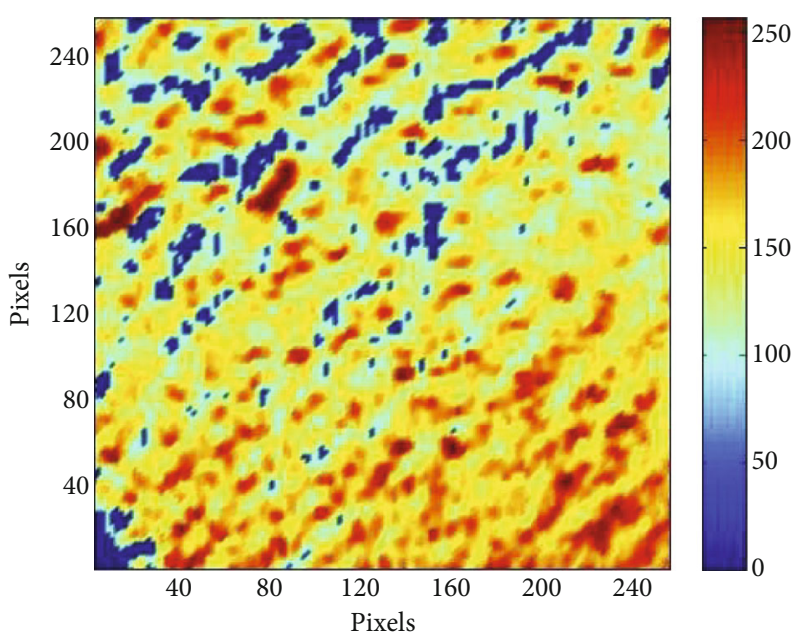

(a)

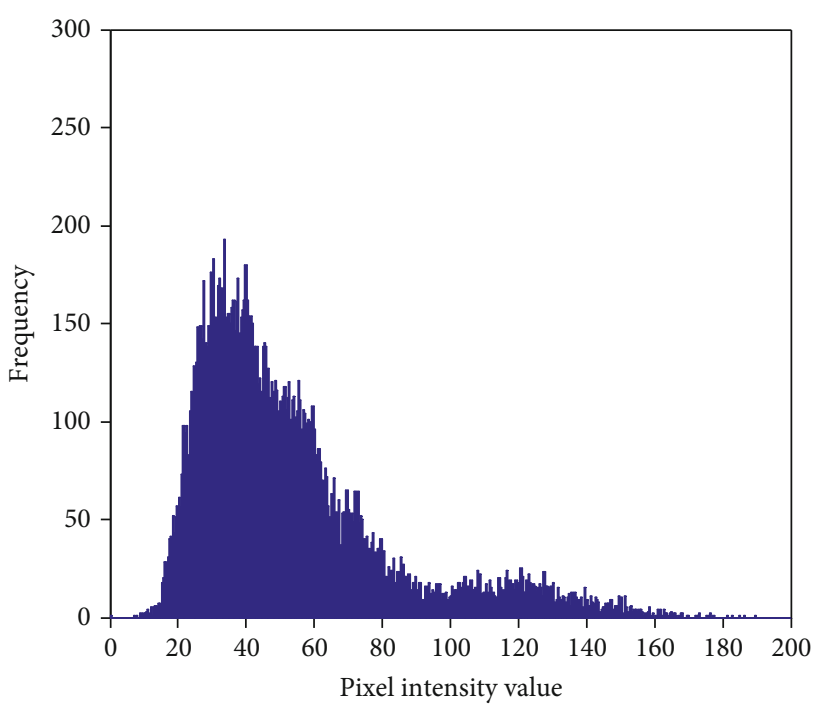

(c)

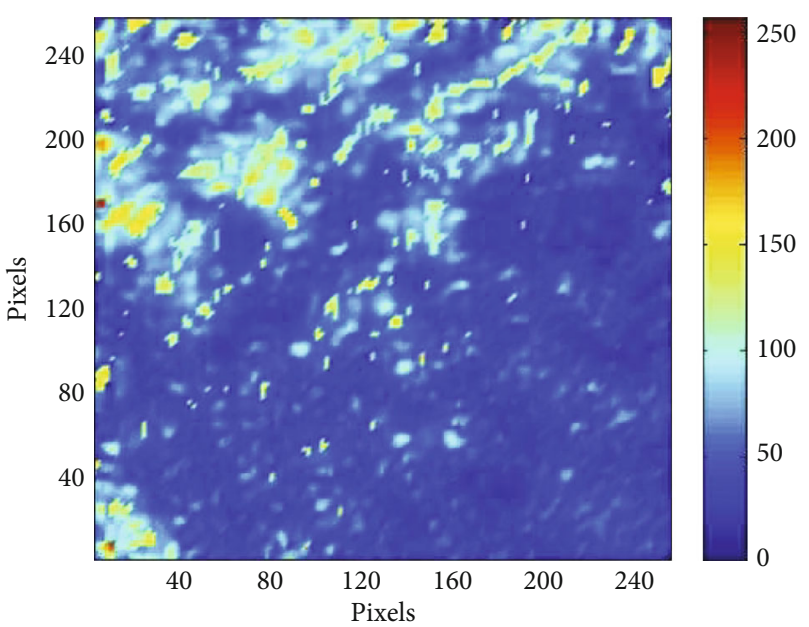

(b)

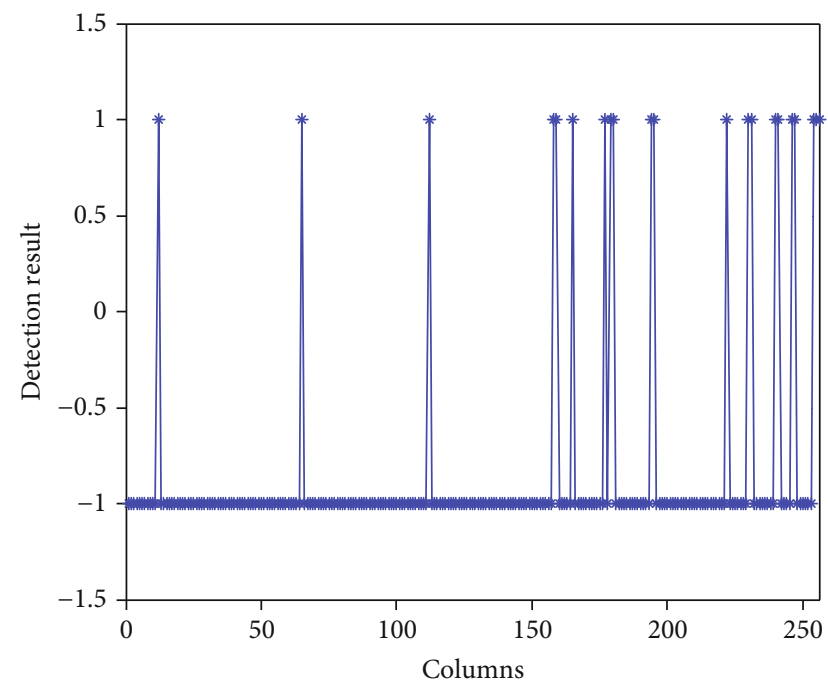

(d)

FIGURE 10: The rainfall detection process of a rain-free radar image: (a) the Cartesian diagram, (b) the radar texture difference map of (a), (c) The frequency distribution of the pixels in (b), and (d) the rainfall detection results of each column in (b).

Here, to ensure that a sufficient number of wavelengths are available for the detection of each column (row) in the radar texture difference map, the method is as follows: when $\pi / 4 \leq \alpha<3 \pi / 4$, the radar texture difference map is detected row-by-row. Otherwise, the radar texture difference map is detected column-by-column. It should be noted that some inconsecutive high pixels, such as ships and floating objects, will exist in the radar image, but they will not affect the detection accuracy of the consecutive pixel method.

\section{Experimental Results and Analysis}

Compared with the rainfall synchronously recorded by the rain gauge, the effectiveness of the proposed method for rainfall detection is certified in this section. Meanwhile, the performance of rainfall detection is analyzed by comparing the proposed method with the original method (in Section 3), the ZPP method, and the SVM-based method. Moreover, the accuracy of the proposed method is analyzed in different wave height environments to validate the applicability of this method.

5.1. Experimental Results. First, the $T_{H}$ is determined. Figure 9 shows the statistical results of the median values of all pixel intensities in each calculated texture difference map. A total of 1200 median values are calculated, and the red horizontal line denotes the average value $(=40.444$ here) of these median values. Thus, the $T_{H}$ is set to 40 .

According to the synchronous measurement value of the rain gauge, a rain-free radar image and a rain-contaminated radar image are randomly selected for rainfall detection by using the WTD method.

Figure 10 shows the rainfall detection process of a rainfree radar image, and the measured value of the rain gauge is $0 \mathrm{~mm}$. The angle $\alpha$ between the wave direction and the north direction is $41^{\circ}$. According to Equation (5), each column of 


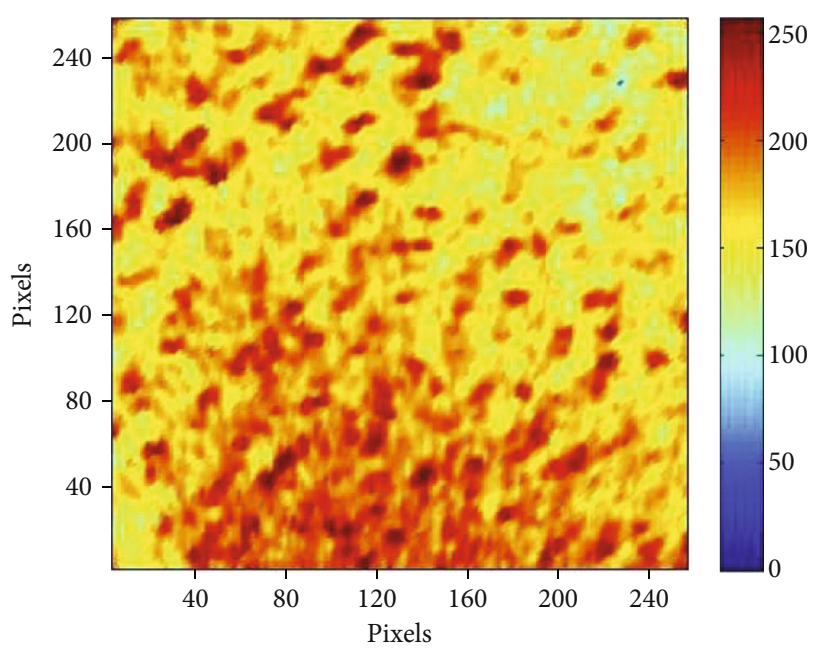

(a)

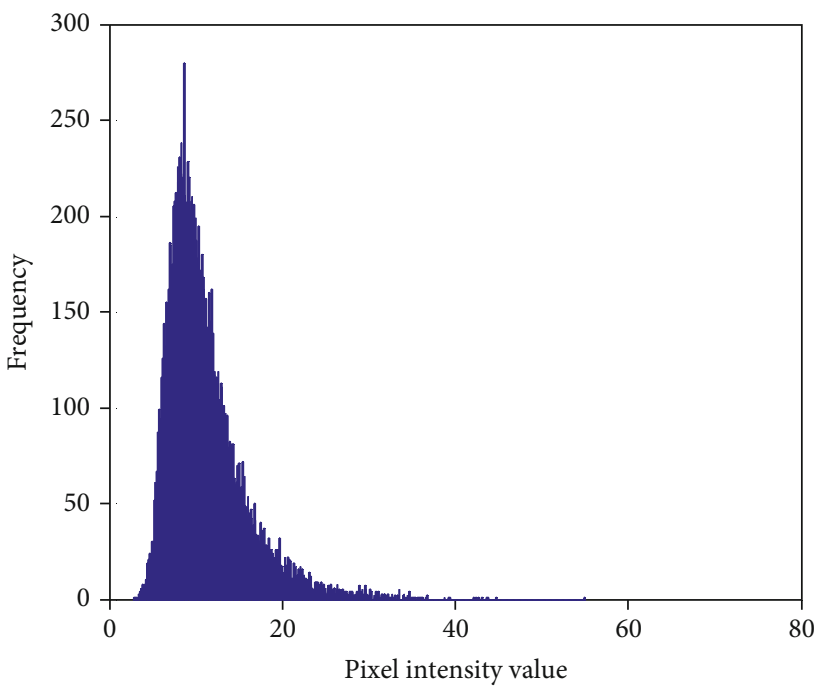

(c)

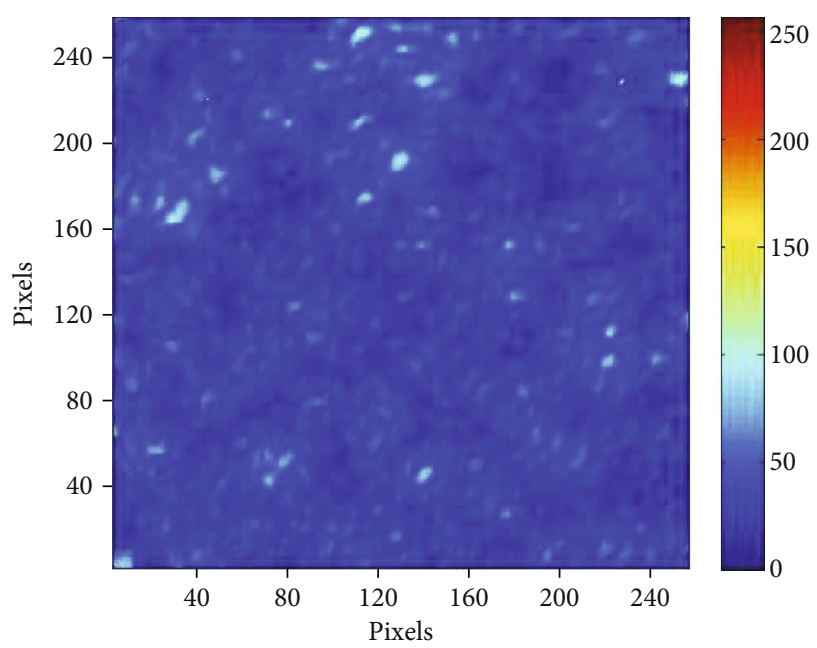

(b)

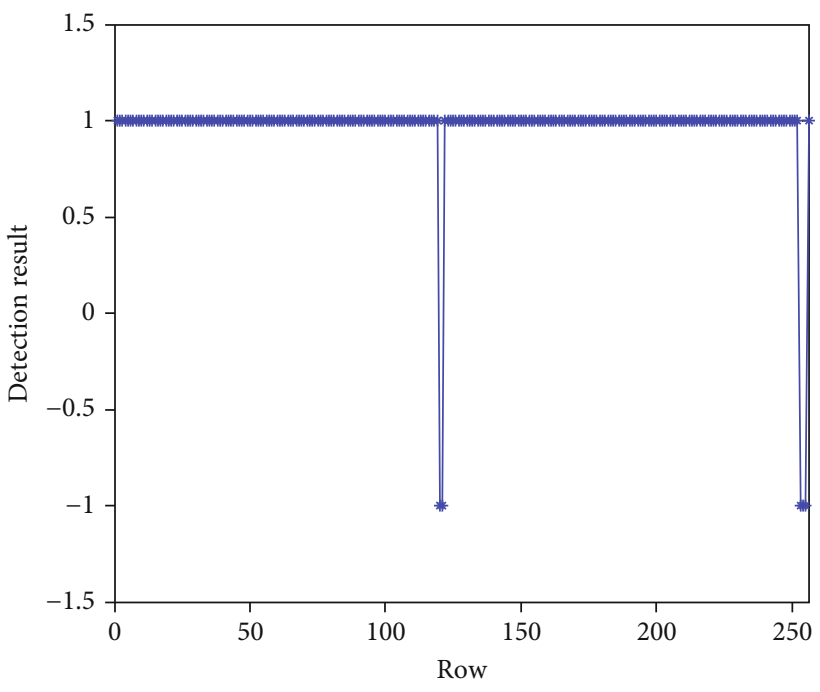

(d)

FIGURE 11: The rainfall detection process of a rain-contaminated radar image: (a) the Cartesian diagram, (b) the radar texture difference map of (a), (c) the frequency distribution of the pixels in (b), and (d) the rainfall detection results of each row in (b).

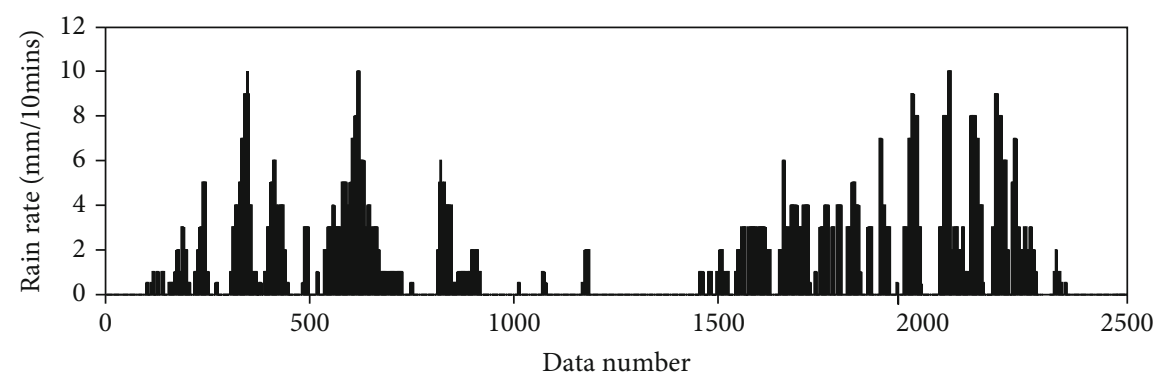

FIGURE 12: The simultaneous rain rate recorded by the rain gauge.

the radar texture difference map is detected, and the value of consecutive pixels $m$ is 53. The Cartesian diagram obtained by coordinate transformation is shown in Figure 10(a). The pixels of Figure 10(a) are calculated by Equation (4) to obtain the corresponding radar texture difference map, as shown in
Figure 10(b). Figure 10(c) shows the frequency distribution of the pixels in Figure 10(b). The horizontal coordinate denotes the pixel intensity value, which also denotes the texture difference value, and the vertical coordinate denotes the frequency of each pixel. Figure 10(d) shows the rainfall detection results of 


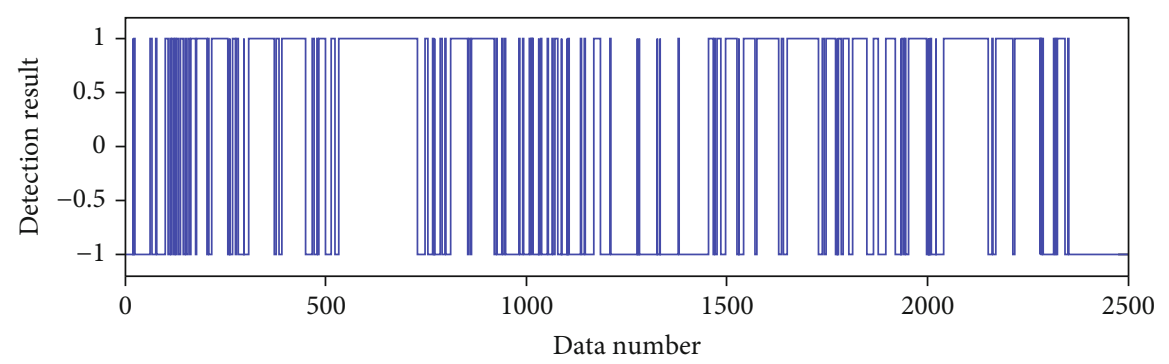

FIGURE 13: The rainfall detection results based on the WTD method.

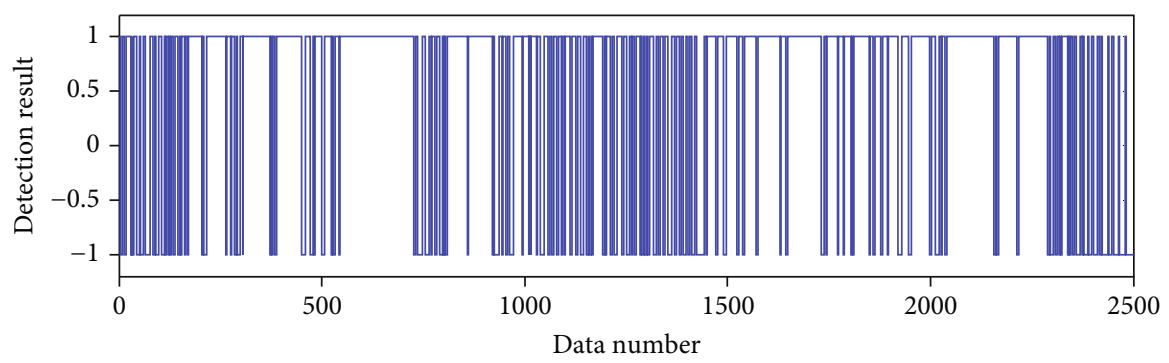

FIGURE 14: The rainfall detection results based on the original method.

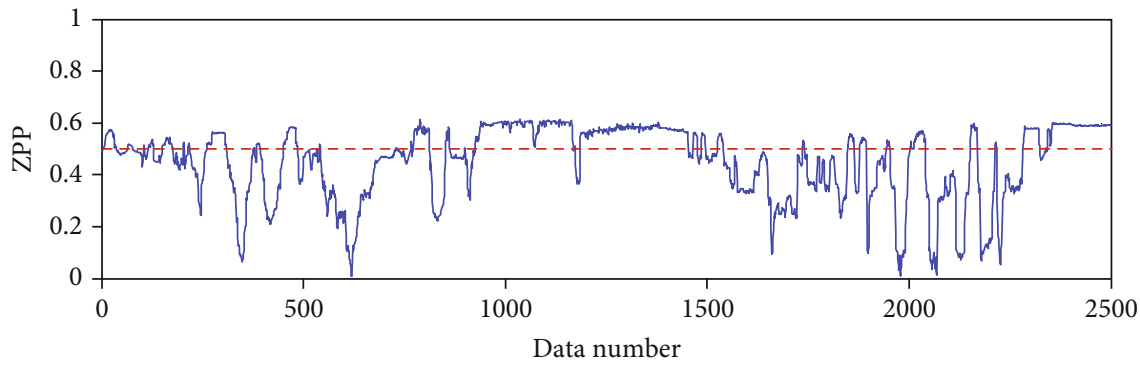

- ZPP

- - Threshold

(a)

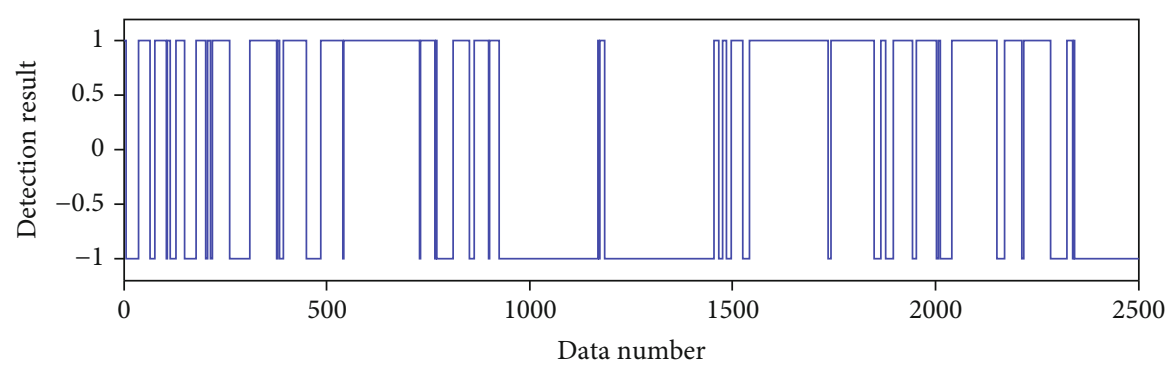

- ZPP

(b)

FIgure 15: (a) The calculated ZPP. (b) The rainfall detection results based on the ZPP method.

each column in Figure 10(b). The horizontal coordinate denotes 256 columns of Figure 10(b), and the vertical coordinate denotes the detection results of each column. When a column is identified as rain-contaminated, the column is labeled as 1 . Otherwise, the column is labeled as -1 . From Figure 10(d), $m$ consecutive results identified as rain-contaminated do not exist in the 256 detection results. Therefore, the selected radar image is detected as a rain-free radar image, and the detection result is correct.

Figure 11 shows the rainfall detection process of a raincontaminated radar image, and the measured value of the rain gauge is $4 \mathrm{~mm}$. The angle $\alpha$ between the wave direction 


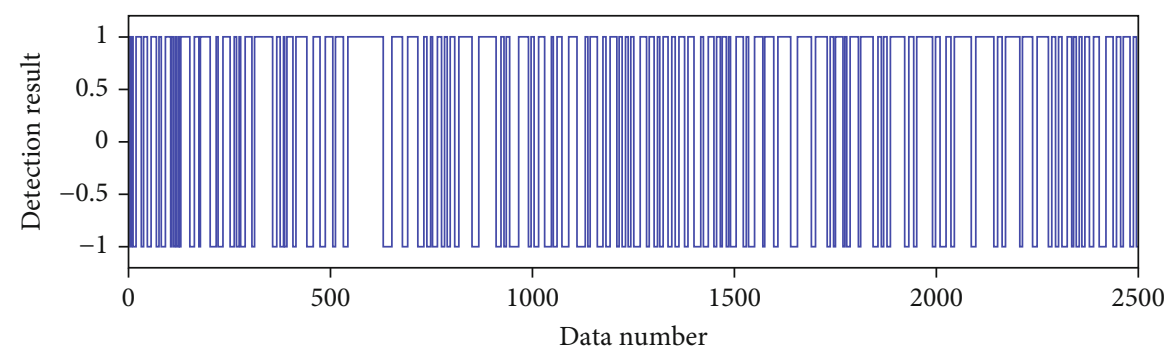

FIgURE 16: The rainfall detection results based on the SVM-based method.

TABLE 2: The comparison of the rainfall detection accuracy.

\begin{tabular}{|c|c|c|c|c|c|}
\hline & \multicolumn{2}{|c|}{ Rain-free image } & \multicolumn{2}{|c|}{ Rain-contaminated image } & \multirow{2}{*}{$\begin{array}{c}\text { Total image } \\
\text { Accuracy }\end{array}$} \\
\hline & Correct number & Accuracy & Correct number & Accuracy & \\
\hline $\mathrm{ZPP}$ & 997 & $79.76 \%$ & 1134 & $90.72 \%$ & $85.24 \%$ \\
\hline The original method & 664 & $53.12 \%$ & 1194 & $95.52 \%$ & $74.32 \%$ \\
\hline The proposed method & 1129 & $90.32 \%$ & 1206 & $96.48 \%$ & $93.4 \%$ \\
\hline
\end{tabular}

TABle 3: The experimental results of the rain-free image data.

\begin{tabular}{lccc}
\hline Wave height $(\mathrm{m})$ & Select number & Correct number & Accuracy \\
\hline 2.0 & 200 & 184 & $92 \%$ \\
1.9 & 200 & 181 & $90.5 \%$ \\
1.8 & 200 & 190 & $95 \%$ \\
1.7 & 200 & 183 & $91.5 \%$ \\
1.6 & 200 & 185 & $92.5 \%$ \\
1.5 & 200 & 183 & $91.5 \%$ \\
1.4 & 200 & 177 & $88.5 \%$ \\
1.3 & 200 & 185 & $92.5 \%$ \\
1.2 & 200 & 181 & $90.5 \%$ \\
1.1 & 200 & 174 & $87 \%$ \\
1.0 & 200 & 171 & $85.5 \%$ \\
0.9 & 200 & 179 & $89.5 \%$ \\
0.8 & 200 & 166 & $83 \%$ \\
0.7 & 200 & 154 & $77 \%$ \\
0.6 & 200 & 131 & $65.5 \%$ \\
0.5 & 200 & 91 & $45.5 \%$ \\
0.4 & 200 & 54 & $27 \%$ \\
0.3 & 200 & 37 & $18.5 \%$ \\
\hline
\end{tabular}

and the north direction is $53^{\circ}$. According to Equation (5), each row of the radar texture difference map is detected, and the value of consecutive pixels $m$ is 50. Figures $11(\mathrm{a})-11(\mathrm{~d})$ show the Cartesian diagram, the radar texture difference map, the frequency distribution histogram, and the rainfall detection results, respectively. From Figure 11(d), most rows of the radar texture difference map are detected as rain-contaminated, and $m$ consecutive results identified as rain-contaminated exist in the 256 detection results. Therefore, the selected radar image is detected as a rain-contaminated radar image, and the detection result is correct.
Then, a large number of radar image data are selected to validate the proposed method. From August 20 to 22, 2013, a total of 2500 radar image data are selected (1250 raincontaminated data and 1250 rain-free data), as shown in Figure 12. The horizontal coordinate denotes the data number. The vertical coordinate denotes the simultaneous rain rate recorded by the rain gauge, and the unit is $\mathrm{mm} / 10 \mathrm{mins}$.

The rainfall detection results based on the WTD method are shown in Figure 13. The horizontal coordinate denotes the data number, and the vertical coordinate denotes the rainfall detection result. When the radar image is detected as raincontaminated, the detection result of this radar image is labeled as 1 . Otherwise, the detection result of this radar image is labeled as -1 . Compared with the simultaneous rain rate in Figure 12, the proposed method has high rainfall detection accuracy. Among the 2500 radar image data, 2335 image data are correctly detected, and the accuracy of rainfall detection is $93.4 \%$. The correct number of the rain-free radar images is 1129 , and the accuracy of rainfall detection is $90.32 \%$. The correct number of the rain-contaminated radar images is 1206 , and the accuracy of rainfall detection is $96.48 \%$. The false alarm rate of the rain-free radar image is higher than that of the rain-contaminated radar image. The reason is that the selected detection area of these rain-free images has small texture differences, which makes these images easier to detect as rain-contaminated images.

To further validate the rainfall detection performance of the proposed method, the rainfall detection result is compared with that of the original method (in Section 3), the ZPP method, and the SVM-based method. Figure 14 shows the rainfall detection results of the original method before improvement [32]. When an azimuthal direction in the texture map is identified as raincontaminated, this direction is regarded as rain-contaminated direction, and the radar image is determined as raincontaminated radar image. Thus, many rain-free radar images are incorrectly detected as rain-contaminated radar images in Figure 14. Among the 2500 radar image data, 1858 image data 


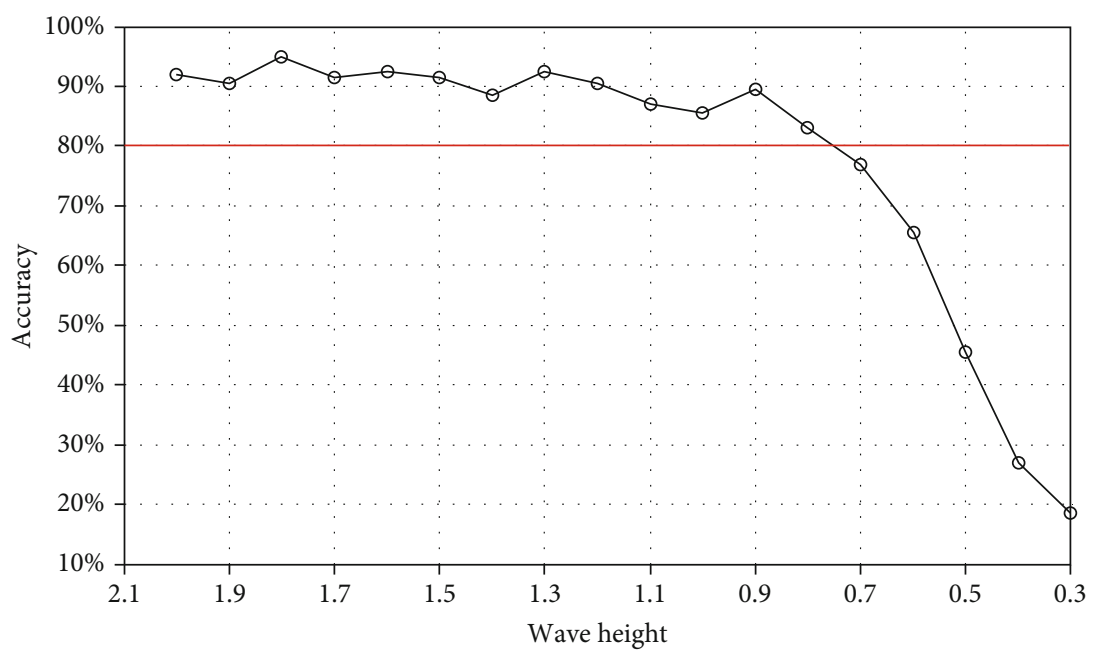

Figure 17: The detection accuracy of the rain-free image data under different wave heights.

TABLE 4: The experimental results of the rain-contaminated image data.

\begin{tabular}{lccc}
\hline Wave height $(\mathrm{m})$ & Select number & Correct number & Accuracy \\
\hline 0.1 & 200 & 200 & $100 \%$ \\
0.2 & 200 & 200 & $100 \%$ \\
0.3 & 200 & 200 & $100 \%$ \\
0.6 & 200 & 196 & $98 \%$ \\
0.7 & 200 & 194 & $97 \%$ \\
0.9 & 200 & 187 & $93.5 \%$ \\
1.1 & 200 & 193 & $96.5 \%$ \\
1.2 & 200 & 189 & $94.5 \%$ \\
1.3 & 200 & 190 & $95 \%$ \\
1.4 & 200 & 193 & $96.5 \%$ \\
1.6 & 200 & 194 & $97 \%$ \\
1.7 & 200 & 191 & $95.5 \%$ \\
2.0 & 200 & 177 & $88.5 \%$ \\
2.1 & 200 & 181 & $90.5 \%$ \\
\hline
\end{tabular}

are correctly detected, and the accuracy of rainfall detection is $74.32 \%$. The correct number of the rain-free radar images is 664 , and the accuracy of rainfall detection is $53.12 \%$. The correct number of the rain-contaminated radar images is 1194 , and the accuracy of rainfall detection is $95.52 \%$.

ZPP is still widely used to detect rain-contaminated radar images because it has the advantages of easy to understand and easy to realize in engineering. In [18], the detection threshold is set to $50 \%$ to determine whether the radar image is contaminated by rainfall. Figure 15(a) shows the curve change of the calculated ZPP. The red dotted line denotes the set $50 \%$ threshold, and the blue solid line denotes the curve change of the calculated ZPP. The calculated ZPP will change with the rainfall, and it will decrease with the increase of the rain rate. When the calculated ZPP is less than the $50 \%$ threshold, the corresponding radar image is determined as the raincontaminated radar image. Otherwise, the radar image is determined as the rain-free radar image.
The rainfall detection results based on the ZPP method are shown in Figure 15(b). When the radar image is detected as rain-contaminated, the detection result of this radar image is labeled as 1 . Otherwise, the detection result of this radar image is labeled as -1 . Among the 2500 radar image data, 2131 image data are correctly detected, and the accuracy of rainfall detection is $85.24 \%$. The correct number of the rain-free radar images is 997 , and the accuracy of rainfall detection is $79.76 \%$. The correct number of the raincontaminated radar images is 1134, and the accuracy of rainfall detection is $90.72 \%$. The false alarm rate of the rain-free radar image is higher than that of the rain-contaminated radar image. The reason is that some rain-free radar images have large wave heights and high pixel intensity, and the calculated ZPP of these images is less than the set threshold. Therefore, these radar images are detected as the raincontaminated radar images.

In addition, reference [23], the SVM-based method is used to detect rain-contaminated radar images in this paper. The data from 201st to 800th are utilized to train the SVM detection model, and the rainfall detection results of the 2500 radar image data are shown in Figure 16. It can be observed from Figure 16 that the detection performance of the SVM-based method is not ideal compared with that of the proposed method, the original method, and the ZPP method. Many rain-free radar images and rain-contaminated radar images are detected incorrectly. The reason is that the training dataset may be less, which makes the performance of the SVM model cannot be well presented. Meanwhile, the SVM-based method for rainfall detection is based on the statistical histogram of the radar image. Since the variation range of the rain rate is relatively large in the selected 2500 radar image data, it is not easy to distinguish the statistical histogram features of the rain-free radar image and the light rain radar image. Therefore, the performance of the SVM-based method for rainfall detection is lower than other methods.

To evaluate the performance of the rainfall detection, the rainfall detection accuracy of the ZPP method, the original method, and the proposed method is compared, as shown in Table 2. From Table 2, the total accuracy of the proposed 


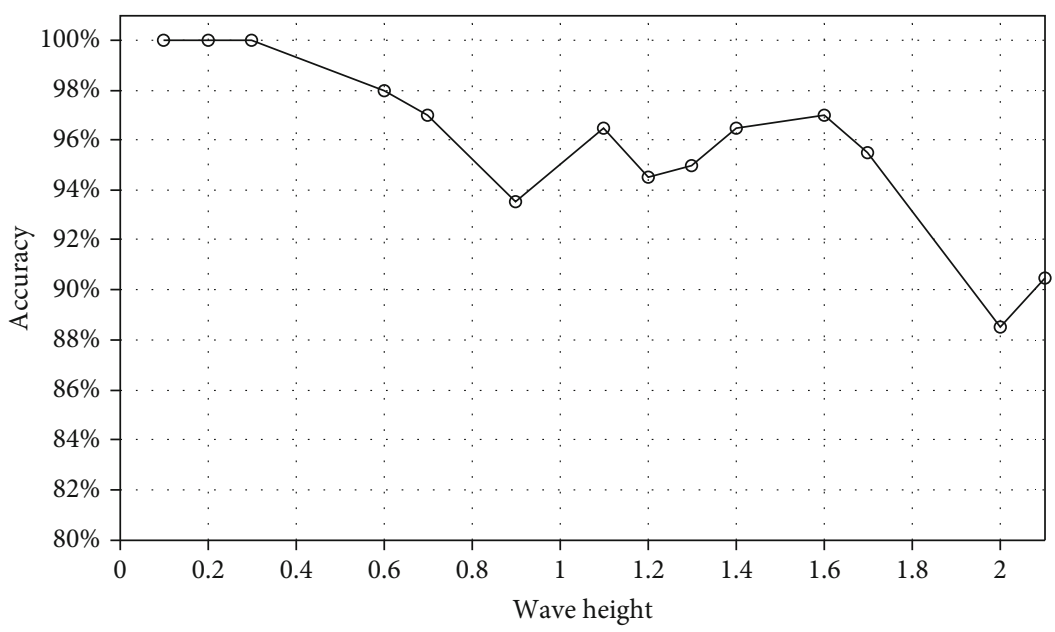

FIGURE 18: The detection accuracy of the rain-contaminated image data under different wave heights.

method is $19.08 \%$ higher than that of the original method before improvement, while it is $8.16 \%$ higher than that of the ZPP method. In particular, the detection accuracy of the rain-free radar image is significantly improved by the proposed method in this paper. It should be noted that the measurement mechanism of the marine radar is different from that of the rain gauge, and the installation position of the rain gauge is far from the radar observation area. Therefore, the detection error exists in the rainfall detection results in this paper.

5.2. The Applicability of the Proposed Method. Since the detection accuracy of the WTD method mainly depends on the wave height environment, the applicability of the proposed method is discussed in this section. According to engineering experience, the waves with wave height greater than $2 \mathrm{~m}$ are regarded as big waves with clear texture. Thus, the applicability experiment starts at a wave height of $2 \mathrm{~m}$. Firstly, 200 rainfree image data are selected under each wave height. The value of the wave height was measured by the buoy, and the measurement accuracy is $0.1 \mathrm{~m}$. Table 3 shows the experimental results, and the relationship between the wave height and the accuracy is shown in Figure 17.

From Figure 17, the red solid line is the accuracy required by the engineering, which is set to $80 \%$, and the variation range of wind speed is $6 \sim 10 \mathrm{~m} / \mathrm{s}$. When the wave height is greater than $0.8 \mathrm{~m}$, the detection accuracy can meet the engineering requirements. When the wave height is less than $0.8 \mathrm{~m}$, the detection accuracy decreases obviously with the decrease of wave height. The reason is that in the low wave environment, the difference of the wave texture is smaller, and the average pixel intensity of the calculated radar texture difference map is small, which makes it easier to detect the rain-free image data as the rain-contaminated image data. When the wave height is $0.6 \mathrm{~m}$, the detection accuracy is still more than $65 \%$. Therefore, when the wave height is greater than or equal to $0.6 \mathrm{~m}$, the proposed method for rainfall detection is effective. When the wave height is greater than or equal to $0.8 \mathrm{~m}$, the proposed method for rainfall detection has high detection accuracy.
Then, 200 rain-contaminated image data are selected under each wave height to discuss the applicability of the proposed method. Here, the rain-contaminated image data is nonzero data measured by the rain gauge. Because the rain-contaminated image data is limited, only the collected rain-contaminated data are statistically analyzed. Table 4 shows the experimental results, and the relationship between the wave height and the accuracy is shown in Figure 18.

Since the rainfall will smooth the waves and reduce the amplitude of the waves in the vertical direction, the wave height of the rain-contaminated image data will be reduced. Here, the maximum wave height of the collected raincontaminated image data is $2.1 \mathrm{~m}$, and the variation range of wind speed is $8 \sim 11 \mathrm{~m} / \mathrm{s}$. From Figure 18, when the wave height is $0.1,0.2$, and $0.3 \mathrm{~m}$, the detection accuracy is $100 \%$. In this wave height environment, the image data is easier to be detected as rain-contaminated image data, and the false alarm rate is high. When the wave height is greater than $0.6 \mathrm{~m}$, the detection accuracy begins to decrease. The reason is that microrain or light rain is not enough to smooth the large waves, and the wave texture difference is still large, which will detect part of the rain-contaminated image data as rainfree image data. When the wave height ranges from $0.6 \mathrm{~m}$ to $2.1 \mathrm{~m}$, the accuracy of the rainfall detection is greater than $88 \%$, which meets the requirements of engineering accuracy. Therefore, in this wave height range, the proposed method for rainfall detection is effective.

It should be noted that the rain-contaminated image data will also be collected in the high wave environment. The reason is that several consecutive waves will be smoothed by rainfall, which is regarded as a complete large wave. For this large wave with smaller amplitude and longer wavelength, the $N$ does not denote the half-wavelength, but less than the half-wavelength. Although the wave texture difference is large in this high wave environment, the average pixel intensity of the calculated texture difference map is small, which makes the image easier to be detected as rain-contaminated radar image.

The experimental results show that when the wave height is greater than $0.6 \mathrm{~m}$, the proposed method in this paper can be used for rainfall detection, and the detection 
accuracy is more than $65 \%$. When the wave height is greater than $0.8 \mathrm{~m}$, the proposed method has high rainfall detection accuracy and meets the requirements of engineering accuracy. Because the obvious wave height exists in the sea in most cases, the method proposed in this paper can be used for rainfall detection in most marine environments.

\section{Conclusions}

In this paper, we propose a WTD method based on the feature that rainfall changes the wave texture information in radar images. Considering the spatial rainfall is uneven, the polar coordinates of the radar image are converted into Cartesian coordinates to detect rainfall. To express the maximum wave difference more accurately, the border points of halfwavelength distance are used to calculate the pixels in the texture difference map. The obtained texture difference map is detected by using the consecutive pixel method, which can detect adaptively with the effect of wind. The data collected from the shore of Haitan Island along the East China Sea are used to validate the proposed method. The results show that the proposed method can not only accomplish the task of rainfall detection but also present a good rainfall detection performance compared with other methods. The total accuracy of the proposed method is $19.08 \%$ higher than that of the original method before improvement, while it is $8.16 \%$ higher than that of the ZPP method. In addition, the image data in different wave height environments are used to validate the applicability of the proposed method. The results demonstrate that the proposed method can be applied to rainfall detection in most marine environments.

Although the experimental results generated using the WTD method are encouraging, it should be noted that the effectiveness of the proposed method needs to be further verified in more wave environments. In addition, since the measurement mechanism of the rain gauge is different from that of the marine radar, the detection error exists in the experimental results in the actual rainfall environment. Moreover, in the future, the data collected from other sites and more radar systems should be utilized to further validate the performance of the proposed method.

\section{Data Availability}

The experimental data used to support the findings of this study are available from the corresponding authors upon request.

\section{Conflicts of Interest}

The author declared that they have no conflicts of interest regarding this work.

\section{Acknowledgments}

This work was supported by the Natural Science Foundation of Jiangsu Province of China under Grant (No. BK20180988) and National Natural Science Foundation of China under Grant (No. 41906154).

\section{References}

[1] T. Brcko, A. Androjna, J. Srse, and R. Boc, "Vessel multiparametric collision avoidance decision model: fuzzy approach," Journal of Marine Science and Engineering, vol. 9, no. $1,2021$.

[2] Z. Chen, Y. He, and B. Zhang, "An automatic algorithm to retrieve wave height from X-band marine radar image sequence," IEEE Transactions on Geoscience and Remote Sensing, vol. 55, no. 9, pp. 5084-5092, 2017.

[3] Y. Wei, Z. Lu, G. Pian, and H. Liu, "Wave height estimation from shadowing based on the acquired $\mathrm{X}$-band marine radar images in coastal area," Remote Sensing, vol. 9, no. 8, 2017.

[4] W. Huang, X. Liu, and E. Gill, "Ocean wind and wave measurements using X-band marine radar: a comprehensive review," Remote Sensing, vol. 9, no. 12, 2017.

[5] Y. Wei, J. K. Zhang, and Z. Lu, "A novel successive cancellation method to retrieve sea wave components from spatio-temporal remote sensing image sequences," Remote Sensing, vol. 8, no. 7, 2016.

[6] Z. Lu, Y. Zhou, and Y. Huang, "Research on correlation in spatial domain to eliminate the co-channel interference of the Xband marine radar," Systems Engineering and Electronics, vol. 39, no. 4, pp. 758-767, 2017.

[7] Y. Li, "Monitoring and mathematical model analysis of dynamic changes in land resources based on SAR sensor image," Journal of Sensors, vol. 2021, Article ID 1661825, 12 pages, 2021.

[8] Z. Lu, B. Lv, L. Li, and S. Guo, "Research on rainfall identification based on the echo differential value from X-band navigation radar image," in 2019 IEEE International Conference on Mechatronics and Automation (ICMA), pp. 2347-2351, Tianjin, China, 2019.

[9] X. Ding, R. Wan, J. Wang, and X. Zheng, "Study on noise suppression for navigation radar images," Ocean Technology, vol. 30, no. 3, pp. 13-16, 2011.

[10] Y. Hao, Y. Tang, and Z. Lu, "The algorithm for noise detection and filtering of the X-band nautical radar image," Remote Sensing for Land \& Resources, vol. 76, pp. 14-17, 2008.

[11] J. C. Nieto-Borge, K. Hessner, P. Jarabo-Amores, and D. MataMoya, "Signal-to-noise ratio analysis to estimate ocean wave heights from X-band marine radar image time series," IET Radar Sonar and Navigation, vol. 2, no. 1, pp. 35-41, 2008.

[12] N. Braun, M. Gade, and P. A. Lange, "The effect of artificial rain on wave spectra and multi-polarisation $\mathrm{X}$ band radar backscatter," International Journal of Remote Sensing, vol. 23, no. 20, pp. 4305-4323, 2002.

[13] F. Xu, X. Li, P. Wang, J. Yang, W. G. Pichel, and Y. Q. Jin, “A backscattering model of rainfall over rough sea surface for synthetic aperture radar," IEEE Transactions on Geoscience and Remote Sensing, vol. 53, no. 6, pp. 3042-3054, 2015.

[14] X. Chen and W. Huang, "Automatic identification of raincontaminated regions in X-band marine radar images," in OCEANS 2019 MTS/IEEE SEATTLE, pp. 1-4, Seattle, WA, USA, 2019.

[15] X. Chen, W. Huang, M. C. Haller, and R. Pittman, "Rain-contaminated region segmentation of X-band marine radar images with an ensemble of SegNets," IEEE Journal of Selected Topics in Applied Earth Observations and Remote Sensing, vol. 14, pp. 141-154, 2021. 
[16] Y. Hao, Y. Tang, and Z. Lu, "Analysis and processing of rainfall observation data in marine radar wave measuring system," Remote Sensing Technology and Application, vol. 23, no. 2, pp. 125-129, 2008.

[17] J. Shen, Y. Li, Y. Dai, and S. Wang, "Identification and suppression of rain interference on X-band radar images," Optics and Precision Engineering, vol. 20, no. 8, pp. 1846-1853, 2012.

[18] B. Lund, H. C. Graber, and R. Romeiser, "Wind retrieval from shipborne nautical X-band radar data," IEEE Transactions on Geoscience and Remote Sensing, vol. 50, no. 10, pp. 38003811, 2012.

[19] Y. Wang and W. Huang, "An algorithm for wind direction retrieval from X-band marine radar images," IEEE Geoscience and Remote Sensing Letters, vol. 13, no. 2, pp. 252-256, 2016.

[20] X. Liu, W. Huang, and E. Gill, "Wind direction estimation from rain-contaminated marine radar data using the ensemble empirical mode decomposition method," IEEE Transactions on Geoscience and Remote Sensing, vol. 55, no. 3, pp. 18331841, 2017.

[21] W. Huang, X. Liu, and E. Gill, “An empirical mode decomposition method for sea surface wind measurements from $\mathrm{X}$ band nautical radar data," IEEE Transactions on Geoscience and Remote Sensing, vol. 55, no. 11, pp. 6218-6227, 2017.

[22] Z. Chen, B. He, B. Zhang, and Y. Ma, "A method to correct the influence of rain on X-band marine radar image," IEEE Access, vol. 5, pp. 25576-25583, 2017.

[23] X. Chen, W. Huang, C. Zhao, and Y. Tian, "Rain detection from X-band marine radar images: a support vector machine-based approach," IEEE Transactions on Geoscience and Remote Sensing, vol. 58, no. 3, pp. 2115-2123, 2020.

[24] Y. Dong and D. Merrett, "Analysis of L-band multi-channel sea clutter," IET Radar Sonar and Navigation, vol. 4, no. 2, pp. 223-238, 2010.

[25] Y. Zheng, Z. Shi, Z. Lu, and W. Ma, "A method for detecting rainfall from X-band marine radar images," IEEE Access, vol. 8, pp. 19046-19057, 2020.

[26] X. Chen and W. Huang, "Identification of rain and lowbackscatter regions in X-band marine radar images: an unsupervised approach," IEEE Transactions on Geoscience and Remote Sensing, vol. 58, no. 6, pp. 4225-4236, 2020.

[27] Z. Lu, L. Sun, and Y. Zhou, "A method for rainfall detection and rainfall intensity level retrieval from X-band marine radar images," Applied Sciences, vol. 11, no. 4, 2021.

[28] R. Shang, P. Peng, F. Shang, L. Jiao, Y. Shen, and R. Stolkin, "Semantic segmentation for SAR image based on texture complexity analysis and key superpixels," Remote Sensing, vol. 12, no. 13, 2020.

[29] J. Lohse, A. Doulgeris, and W. Dierking, "Incident angle dependence of Sentinel-1 texture features for sea ice classification," Remote Sensing, vol. 13, no. 4, 2021.

[30] W. Zhai, C. Huang, and W. Pei, "Building damage assessment based on the fusion of multiple texture features using a single post-earthquake PolSAR image," Remote Sensing, vol. 11, no. $8,2019$.
[31] Q. Chen, H. Yang, L. Li, and X. Liu, "A novel statistical texture feature for SAR building damage assessment in different polarization modes," IEEE Journal of Selected Topics in Applied Earth Observations and Remote Sensing, vol. 13, pp. 154-165, 2020.

[32] W. Huang, Y. Liu, and E. Gill, "Texture-analysis-incorporated wind parameters extraction from rain-contaminated X-band nautical radar images," Remote Sensing, vol. 9, no. 2, 2017. 